\title{
EDITORIAL
}

\section{Safety of resistance training in the elderly}

\author{
Michael Sagiv
}

Received: 15 February 2009 / Accepted: 17 February 2009/Published online: 20 March 2009

(C) European Group for Research into Elderly and Physical Activity (EGREPA) 2009

Disuse of skeletal muscle is often cited as a potentially reversible cause of ill health in elderly people. It can lead to a serious decline in functional health and increases the risk of outpatient care, hospitalization, nursing home admission, and death. The loss of muscle mass accounts for most of the observed loss of strength. The loss of muscle tissue is due to a decrease in the number of muscle fibers and atrophy of the type II muscle fibers. The subsequent decline in strength reduces the capacity to carry out basic activities of daily life and puts people at risk for falls, impaired mobility, and dependence [1]. Although sedentary lifestyle and aging itself may contribute to muscle weakness and loss of skeletal-muscle mass, that conditions are potentially preventable or reversible with targeted interventions [2]. It has been suggested that high-intensity resistance exercise training is a feasible and effective means of counteracting muscle weakness and physical frailty in elderly people [3].

The principal goal of resistive training in the elderly is to restore physical, psychological, and vocational function. However, resistance exercise has previously been regarded as thermodynamically hazardous due to exaggerated blood pressure responses causing higher double pressure products leading to more ischemic events. Traditionally, training programs for older subjects have emphasized lower extremity aerobic exercise (i.e., walking, stationary cycle ergometer, and stair climbing).

Various vocational, recreational, and other activities of daily living actually resemble heavy resistance exercise

\footnotetext{
M. Sagiv $(\bowtie)$

Biogenetic Laboratory,

The Zinman College of Physical Education and Sport Sciences

at Wingate,

Netanya 42902, Israel

e-mail: sagiv@wincol.ac.il
}

more than aerobic exercise. Many elderly people lack the ability to perform such common activities as carrying groceries or luggage or doing yard work. Therefore, it is important to recognize that the elderly require a minimum level of muscle strength for activities of daily living. Evidence has been accumulated, which suggests that resistive exercise training may be less hazardous than was once presumed. The benefits of resistance training for the elderly are improved muscle strength and endurance, enhanced bone mineral density, weight reduction, maintaining an improved self-image, and gaining confidence.

Resistance training can produce a slight increase in aerobic capacity, which is associated with the increase in muscle mass. In the elderly, exercise capacity can be limited by leg fatigue resulting in termination of exercise without coexisting evidence of cardiorespiratory limitation. Studies conducted on the elderly have demonstrated increased strength with enhanced treadmill performance in circuit weight training trainees. Therefore, those with weak leg muscles will gain additional benefits if conventional endurance training is combined with resistance training.

Electrocardiographic and direct arterial blood pressure responses during single-arm and single and double-leg lifting at $20 \%, 40 \%, 60 \%$, and $80 \%$ of one-repetition maximum (1-RM) did not reveal any clinically significant ST-segment depression, angina, or ventricular arrhythmias, except when subject were working against high resistance: $80 \%$ of $1-\mathrm{RM}$, double pressure product values exceeded those attained during cycle ergometer testing at $85 \%$ of maximal aerobic capacity. Thus, light to moderate resistive training exercise can be considered safe for low-risk cardiac patients. Even the risk of developing compromised left ventricular function is less during a workout against weight resistance compared to that in conventional clinical aerobic exercise test [7]. 
Circuit weight training is an approach for strength development especially designed for the elderly. It incorporates a series of selected resistive training exercises that are performed in a sequence. With circuit weight training, one performs approximately ten to 12 repetitions using about $50-55 \%$ of $1-\mathrm{RM}$ on specialized weight machines. The individual moves from one weight machine to another with a short rest $(30-60 \mathrm{~s})$ between stations. The fact that circuit weight training can improve strength, body composition, and, in a modest way, cardiorespiratory endurance makes this form of exercise appealing for training programs for elderly [5].

A large amount of evidence on the safety and efficacy of this kind of resistive training in the elderly has been gathered in the past two decades [4]. Blood pressure measurements in elderly subjects collected before, during, and after circuit weight training demonstrated a slight increase or no change compared to resting values. In comparison to blood pressure values measured during dynamic exercise at $85 \%$ of peak oxygen uptake, circuit weight training blood pressure values were lower or slightly higher than those achieved at $85 \%$ of peak oxygen uptake [6].

Weight training programs result in an increase in muscle strength. A stronger musculature may reduce the relative stress imposed by activities of daily living. Thus, resistance exercise can contribute to better health by preventing musculoskeletal disorders, helping to maintain desirable body composition and improving self-image and selfefficacy. Also, engaging in resistance exercise may reduce some risk factors for coronary artery disease. Improvements are seen in physical fitness and quality of life, as well as in the modification of classical risk factors for coronary artery disease. In addition, the aging musculoskeletal system retains its responsiveness to progressive resistance training, and most importantly, the correction of disuse is accompanied by a significant improvement in the level of functional mobility and the overall ability to conduct activities safety.

\section{References}

1. Aniansson A, Sperling L, Rundgren A et al (1983) Muscle function in 75-year-old men and women. Scand J Rehabil Med Suppl 9:92102

2. Fiatarone MA, Evans WJ (1993) The etiology and reversibility of muscle dysfunction in the aged. J Gerontol 48:77-83

3. Fiatarone MA, O'Neill EF, Ryan ND et al (1994) Exercise training and dietary supplementation in the elderly. $\mathrm{N}$ Engl $\mathrm{J}$ Med 330:1769-1775. doi:10.1056/NEJM199406233302501

4. Karlsdottir AE, Foster C, Porcari JP et al (2002) Hemodynamic responses during aerobic and resistance exercise. J Cardiopulm Rehabil 22:170-177. doi:10.1097/00008483-200205000-00008

5. Monteiro AG, Alveno DA, Prado M et al (2008) Acute physiological responses to different circuit training protocols. J Sports Med Phys Fitness 48:438-442

6. Sparling PB, Cantwell JD, Dolan CM et al (1990) Strength training in a cardiac rehabilitation program: a six-month follow-up. Arch Phys Med Rehabil 71:148-152

7. Sagiv M, Sagiv A, Ben-Sira D et al (1997) Effects of chronic overload training and aging on left ventricular systolic function. Gerontology 43:307-315

\section{Acknowledgement to Reviewers}

The Editors would like to thank the following reviewers for their most helpful support during the year 2008:

Ayalon, Mosh, Natanya, Israel

Ben-Sira, David Goldhammer Ehud, Haifa, Israel

Boecker, Henning, Bonn, Germany

Chen, Yamin, Natanya, Israel

Coppenolle, Herman Van, Leuven, Belgium

Duarte, Jose, Porto, Portugal

Konczak, Jürgen, Minneapolis, USA

Messerli Buergy, Nadine, London, UK

Netz, Yael, Natanya, Israel

Nir, Eynon, Natanya, Israel

Oliveira, Jose, Porto, Portugal

Sagiv, Moran, Natanya, Israel

Schlag, Bernhard, Dresden, Germany

Vogelaere, Peter, Avora, Portugal

Winkler, Christian, Freiburg, Germany

Woll, Alexander, Konstanz, Germany 